\title{
Multiplicity and the Lojasiewicz exponent
}

\author{
by S. Spodzieja (Łódź)
}

\begin{abstract}
We give a formula for the multiplicity of a holomorphic mapping $f$ : $\mathbb{C}^{n} \supset \Omega \rightarrow \mathbb{C}^{m}, m>n$, at an isolated zero, in terms of the degree of an analytic set at a point and the degree of a branched covering. We show that calculations of this multiplicity can be reduced to the case when $m=n$. We obtain an analogous result for the local Łojasiewicz exponent.
\end{abstract}

Introduction. Achilles, Tworzewski and Winiarski [ATW] introduced a definition of the improper isolated intersection multiplicity of analytic sets. Tworzewski $[\mathrm{Tw}]$ generalized this definition to any improper intersections of analytic sets. This biholomorphic invariant plays an important role in the theory of singularities of analytic sets. The above multiplicity leads to the definition of multiplicity of a holomorphic mapping at a zero of this mapping (see $[\mathrm{TW}]$ ). We study the multiplicity $i_{0}(f)$ of a holomorphic mapping $f$ : $\Omega \rightarrow \mathbb{C}^{m}$ at an isolated zero $0 \in \Omega$, where $\Omega \subset \mathbb{C}^{n}$ is an open set and $m>n$.

The main result of this paper is a formula for $i_{0}(f)$ (Theorem 1.2). Namely, for a sufficiently small neighbourhood $D \subset \Omega$ of 0 ,

$$
i_{0}(f)=\left.\operatorname{deg} f\right|_{D} \operatorname{deg}_{0} f(D),
$$

where $\left.f\right|_{D}: D \rightarrow f(D)$ is a branched covering (i.e. a $*$-covering in the sense of $\left[\mathrm{E}_{2}\right]$, V.7.2). The crucial role in the proof of Theorem 1.2 is played by the reduction of the calculations of the multiplicity of $f$ at an isolated zero to the case $m=n$ (see Theorem 1.1). We obtain this result by the composition of $f$ with a linear mapping. From Theorem 1.1 it follows that the multiplicity $i_{0}(f)$ depends on the ideal generated by the components of $f$ only (see Corollary 1.1, cf. [ATW], Proposition 6.1). Additionally we obtain an estimate from below of the multiplicity of a holomorphic mapping at an isolated zero in terms of the orders of the components of the mapping (see Corollary 1.2).

2000 Mathematics Subject Classification: Primary 32S99; Secondary 32B99.

Key words and phrases: holomorphic mapping, local Łojasiewicz exponent, multiplicity. This research was partially supported by KBN Grant 2 P03A 00718. 
We apply considerations analogous to those in Theorem 1.1 to the local Łojasiewicz exponent. We show that the calculation of the Eojasiewicz exponent of $f$ can be reduced to the case $m=n$ (see Theorem 2.1). In the case $m=n$ relations between the above mentioned biholomorphic invariants are well known (see $\left[\mathrm{E}_{1}\right]$, [D'A], [CL], $\left[\mathrm{P}_{1}\right]-\left[\mathrm{P}_{3}\right],[\mathrm{C}]$, [Te], [LJT], [KL]). We show that some of them also hold in the case $m>n$ (see Proposition 3.1).

1. The multiplicity of isolated zeros of holomorphic mappings. In the whole paper we will assume that $\Omega \subset \mathbb{C}^{n}$ is a neighbourhood of $0 \in \mathbb{C}^{n}$ and

$$
f=\left(f_{1}, \ldots, f_{m}\right): \Omega \rightarrow \mathbb{C}^{m}
$$

is a holomorphic mapping having an isolated zero at $0, m \geq n$.

By the multiplicity of $f$ at 0 we mean the improper intersection multiplicity $i$ (graph $\left.f \cdot\left(\mathbb{C}^{n} \times\{0\}\right) ;(0,0)\right)$ of the graph $f$ and $\mathbb{C}^{n} \times\{0\}$ at the point $(0,0) \in \mathbb{C}^{n} \times \mathbb{C}^{m}$ (see $[\mathrm{ATW}]$ ) and we denote it by $i_{0}(f)$. If $m=n$ then we have $i_{0}(f)=\mu_{0}(f)$, where $\mu_{0}(f)$ denotes the covering multiplicity of $f$ at 0 .

We shall show that considerations of multiplicity at an isolated zero of a holomorphic mapping $f: \mathbb{C}^{n} \supset \Omega \rightarrow \mathbb{C}^{m}$ can be reduced to the case when $m=n$.

Denote by $\mathbf{L}(m, k)$ the set of all nonsingular linear mappings $\mathbb{C}^{m} \rightarrow \mathbb{C}^{k}$, where for $k=0$ we put $\mathbb{C}^{k}=\{0\}$.

In what follows we will write "the generic $x \in A$ " instead of "there exists an algebraic set $V$ such that $A \backslash V$ is a dense subset of $A$ and $x \in A \backslash V$ ".

Let $f: \Omega \rightarrow \mathbb{C}^{m}$ be as above.

Theorem 1.1. Let $n \leq k \leq m$. Then for any $L \in \mathbf{L}(m, k)$ such that the point 0 is an isolated zero of $L \circ f: \Omega \rightarrow \mathbb{C}^{k}$ we have

$$
i_{0}(f) \leq i_{0}(L \circ f) \text {. }
$$

Moreover, for the generic $L \in \mathbf{L}(m, k)$, the point 0 is an isolated zero of $L \circ f$ and

$$
i_{0}(f)=i_{0}(L \circ f) .
$$

The proof of this theorem will be preceded by two definitions and a lemma.

Let $V$ be an analytic set in a neighbourhood $D \subset \mathbb{C}^{N}$ of $0 \in \mathbb{C}^{N}, 0 \in V$. By $C_{0}(V)$ we denote the tangent cone to $V$ at 0 in the sense of Whitney ([W], p. 510). The tangent cone $C_{0}(V)$ is clearly a closed cone with vertex 0 . Moreover, it is an algebraic set of dimension $\operatorname{dim}_{0} V$. If 0 is a simple point of $V$, then $C_{0}(V)$ is the tangent space $T_{0}(V)$ to $V$ at 0 .

Let $V, W$ be analytic sets in a neighbourhood $D \subset \mathbb{C}^{N}$ of $0 \in \mathbb{C}^{N}$, $0 \in V \cap W$. The relative tangent cone $C_{0}(V, W)$ of the sets $V, W$ at 0 is 
defined as the set of $v \in \mathbb{C}^{N}$ such that there exist sequences $\left\{x_{j}\right\} \subset V$, $\left\{y_{j}\right\} \subset W$ and $\left\{t_{j}\right\} \subset \mathbb{C}$ satisfying

$$
x_{j} \rightarrow 0, \quad y_{j} \rightarrow 0, \quad t_{j}\left(x_{j}-y_{j}\right) \rightarrow v \quad \text { as } j \rightarrow \infty .
$$

It is easy to see that the relative tangent cone $C_{0}(V, W)$ is clearly a closed cone with vertex 0 .

LEMMA 1.1. Let $\Omega \subset \mathbb{C}^{n}$ be a neighbourhood of $0 \in \mathbb{C}^{n}$ such that $\bar{\Omega}$ is a compact set. Let $f$ be a holomorphic mapping in some neighbourhood of $\bar{\Omega}$ with values in $\mathbb{C}^{m}$ such that $f^{-1}(0) \cap \bar{\Omega}=\{0\}$ and $f(\Omega)$ generates an irreducible analytic germ at $0 \in \mathbb{C}^{m}$. Then

$$
C_{(0,0)}\left(\mathbb{C}^{n} \times\{0\}, \operatorname{graph} f\right)=\mathbb{C}^{n} \times C_{0}(f(\Omega)) .
$$

Proof. Take any sequences $\left\{x_{j}\right\} \subset \Omega,\left\{\left(x_{j}^{\prime}, f\left(x_{j}^{\prime}\right)\right)\right\} \subset$ graph $f$ and $\left\{t_{j}\right\} \subset \mathbb{C}$ such that $x_{j} \rightarrow 0$ and $x_{j}^{\prime} \rightarrow 0$ as $j \rightarrow \infty$. If

$$
t_{j}\left(x_{j}^{\prime}-x_{j}, f\left(x_{j}^{\prime}\right)\right) \rightarrow v \in \mathbb{C}^{n+m},
$$

then obviously $v \in \mathbb{C}^{n} \times C_{0}(f(\Omega))$.

Take any $v=(x, y) \in \mathbb{C}^{n} \times C_{0}(f(\Omega))$. Assume that $y=0$, and $x \neq 0$. Let $x_{j}^{\prime}=(1 / j) x$. Then there exists $\left\{t_{j}\right\} \subset \mathbb{C}$ such that $t_{j} \rightarrow \infty$ and $t_{j} f\left(x_{j}^{\prime}\right) \rightarrow 0$. Let $x_{j}=x_{j}^{\prime}-\left(1 / t_{j}\right) x$. Then obviously $x_{j} \rightarrow 0$ and $(3)$ holds. Thus $v \in C_{0}\left(\mathbb{C}^{n} \times\{0\}\right.$, graph $\left.f\right)$. Let now $y \neq 0$. Then there exist sequences $\left\{x_{j}^{\prime}\right\} \subset \mathbb{C}^{n}$ and $\left\{t_{j}\right\} \subset \mathbb{C}$ such that

$$
f\left(x_{j}^{\prime}\right) \rightarrow 0, \quad t_{j} f\left(x_{j}^{\prime}\right) \rightarrow y .
$$

Hence, $t_{j} \rightarrow \infty$ and, by the assumptions, $x_{j}^{\prime} \rightarrow 0$. Taking $x_{j}$ as above we obtain (3) and so, $v \in C_{0}\left(\mathbb{C}^{n} \times\{0\}\right.$, graph $\left.f\right)$. This ends the proof.

Proof of Theorem 1.1. If $m=n$ or $k=m$, then the assertion is obvious. Assume that $m>k \geq n$.

Consider the case $k=n$. Let $L \in \mathbf{L}(m, n)$ be such that 0 is an isolated point of $(L \circ f)^{-1}(0)$. Let

$$
H=\mathbb{C}^{n} \times \operatorname{ker} L .
$$

Then from the definitions of multiplicity of improper and proper intersections we have

$$
i_{0}(f)=i\left(\operatorname{graph} f \cdot\left(\mathbb{C}^{n} \times\{0\}\right) ;(0,0)\right) \leq i(\operatorname{graph} f \cdot H ;(0,0))=\mu_{0}(L \circ f),
$$

which gives (1) in this case.

We now prove the second part of the theorem. Since the considerations have a local character and 0 is an isolated zero of $f$, we may assume that $\bar{\Omega}$ is a compact set, $f$ is a holomorphic mapping in a neighbourhood of $\bar{\Omega}$, $f^{-1}(0) \cap \bar{\Omega}=\{0\}$ and $W=f(\Omega)$ generates an irreducible analytic germ at $0 \in \mathbb{C}^{m}$. Let $C_{0}(W)$ be the tangent cone of $W$ at 0 . Then $\operatorname{dim} C_{0}(W)=$ $\operatorname{dim} W$. So, for the generic $L \in \mathbf{L}(m, n)$, by Corollary of $\left[\mathrm{E}_{2}\right]$, VII.11.8, we 
obtain $C_{0}(W) \cap \operatorname{ker} L \subset\{0\}$, thus 0 is an isolated point of $(L \circ f)^{-1}(0)$. From Lemma 1.1 we get

$$
C_{(0,0)}\left(\mathbb{C}^{n} \times\{0\}, \text { graph } f\right)=\mathbb{C}^{n} \times C_{0}(W) .
$$

Thus, using Theorem 4.4 of [ATW], we easily see that for any $L \in \mathbf{L}(m, n)$ such that $C_{0}(W) \cap \operatorname{ker} L=\{0\}$,

$$
i\left(\operatorname{graph} f \cdot \mathbb{C}^{n} \times\{0\} ;(0,0)\right)=i(\operatorname{graph} f \cdot H ;(0,0)),
$$

where $H=\mathbb{C}^{n} \times \operatorname{ker} L$. So,

$$
i_{0}(f)=\mu_{0}(L \circ f) .
$$

Since the set $\left\{L \in \mathbf{L}(m, n): C_{0}(W) \cap \operatorname{ker} L=\{0\}\right\}$ is a Zariski open and dense subset of $\mathbf{L}(m, n)$, we obtain (2) for the generic $L \in \mathbf{L}(m, n)$.

Now consider the last case $n<k<m$. Take any $L \in \mathbf{L}(m, k)$ such that 0 is an isolated zero of $L \circ f: \Omega \rightarrow \mathbb{C}^{k}$. Then, by the first part of the proof, we have

$$
i_{0}(L \circ f)=\min _{\widetilde{L} \in \mathbf{L}(k, n)} \mu_{0}(\widetilde{L} \circ L \circ f) \geq \min _{\bar{L} \in \mathbf{L}(m, n)} \mu_{0}(\bar{L} \circ f)=i_{0}(f) .
$$

This gives (1) in this case.

Let $\mathcal{U} \subset \mathbf{L}(m, n)$ be the set of all $M \in \mathbf{L}(m, n)$ such that $i_{0}(M \circ f)$ $=i_{0}(f)$. Then, by the first part of the proof, the set

$$
\mathcal{U}^{\prime}=\left\{L=\left(M, M^{\prime}\right) \in \mathbf{L}(m, k): M \in \mathcal{U}, M^{\prime} \in \mathbf{L}(m, k-n)\right\}
$$

contains a Zariski open subset of $\mathbf{L}(m, k)$. Moreover, for any $L=\left(M, M^{\prime}\right)$ $\in \mathcal{U}^{\prime}$, taking $\widetilde{L}\left(y_{1}, \ldots, y_{k}\right)=\left(y_{1}, \ldots, y_{n}\right)$ we obtain

$$
i_{0}(f) \leq i_{0}(L \circ f) \leq i_{0}\left(\widetilde{L} \circ\left(M, M^{\prime}\right) \circ f\right)=i_{0}(M \circ f)=i_{0}(f) .
$$

This gives (2) for any $L \in \mathcal{U}^{\prime}$ and ends the proof in this case.

This ends the proof of Theorem 1.1.

Denote by $\mathcal{O}_{0}$ the ring of germs of holomorphic functions at $0 \in \mathbb{C}^{n}$, and by $\mathfrak{m}_{0}$ the maximal ideal of $\mathcal{O}_{0}$.

Theorem 1.1 and Theorem 5.2 of [ATW] yield

COROLlary 1.1. The multiplicity $i_{0}(f)$ is a biholomorphic invariant and depends on the ideal $\left(f_{1}, \ldots, f_{m}\right) \mathcal{O}_{0}$ only.

Proof. The first part immediately follows from the fact that the intersection multiplicity of analytic sets is a biholomorphic invariant (see [ATW], Theorem 5.2). To show the second part, observe that for any functions $\beta_{i, j}$, $i, j=1, \ldots, m$, holomorphic in a neighbourhood of 0 such that the matrix 
$\left[\beta_{i, j}\right]$ is invertible, we have

$$
i_{0}(f)=i_{0}(\widetilde{f}),
$$

where $\widetilde{f}=\left[\beta_{i, j}\right] f$. Indeed, since $\left[\beta_{i, j}\right]$ is invertible, there exist neighbourhoods $U, D \subset \mathbb{C}^{n} \times \mathbb{C}^{m}$ such that

$$
\xi: U \ni(x, y) \mapsto\left(x,\left[\beta_{i, j}\right] y\right) \in D
$$

is a biholomorphism. Moreover $\xi\left(U \cap\left(\mathbb{C}^{n} \times\{0\}\right)\right)=D \cap\left(\mathbb{C}^{n} \times\{0\}\right)$ and $\xi(U \cap \operatorname{graph} f)=D \cap \operatorname{graph} \widetilde{f}$. Thus, by Theorem 5.2 of $[\mathrm{ATW}]$, we have the announced observation.

Let $g_{1}, \ldots, g_{k}$ be holomorphic functions about 0 such that

$$
\left(f_{1}, \ldots, f_{m}\right) \mathcal{O}_{0}=\left(g_{1}, \ldots, g_{k}\right) \mathcal{O}_{0} .
$$

From Theorem 1.1, we see that

$$
i_{0}(f)=i_{0}\left(f_{1}, \ldots, f_{m}, 0, \ldots, 0\right),
$$

so, from the above observation,

$$
i_{0}(f)=i_{0}\left(f_{1}, \ldots, f_{m}, g_{1}, \ldots, g_{k}\right)=i_{0}\left(0, \ldots, 0, g_{1}, \ldots, g_{k}\right)=i_{0}\left(g_{1}, \ldots, g_{k}\right) .
$$

This ends the proof.

REMARK 1.1. Theorem 1.1 yields $\left(\mathfrak{m}_{0}\right)^{i_{0}(f)} \subset\left(f_{1}, \ldots, f_{m}\right) \mathcal{O}_{0}$.

Indeed, by Theorem 1.1, there exists $L \in \mathbf{L}(m, n)$ such that $i_{0}(f)=$ $\mu_{0}(L \circ f)$. Let $\mathcal{J}$ be the ideal in $\mathcal{O}_{0}$ generated by the components of the mapping $L \circ f$. It is well known that $\left(\mathfrak{m}_{0}\right)^{\mu_{0}(L \circ f)} \subset \mathcal{J}$. Since $\mathcal{J} \subset\left(f_{1}, \ldots, f_{m}\right) \mathcal{O}_{0}$, we obtain the assertion.

Let $m \geq k$. Denote by $\Delta(m, k)$ the set of all linear mappings $L=$ $\left(L_{1}, \ldots, L_{k}\right) \in \mathbf{L}(m, k)$ of the form

$$
L_{i}\left(y_{1}, \ldots, y_{m}\right)=y_{i}+\sum_{j=k+1}^{m} \alpha_{i, j} y_{j}, \quad i=1, \ldots, k,
$$

where $\alpha_{i, j} \in \mathbb{C}$.

From Theorem 1.1, it is easy to deduce

Proposition 1.1. For the generic $L=\left(L_{1}, \ldots, L_{n}\right) \in \Delta(m, n)$, the point 0 is an isolated zero of $L \circ f: \Omega \rightarrow \mathbb{C}^{n}$ and

$$
i_{0}(f)=i_{0}(L \circ f)=\mu_{0}(L \circ f) .
$$

Moreover, if $k_{j}=$ ord $f_{j}, k_{1} \leq \ldots \leq k_{m}$, then

$$
\operatorname{ord}\left(L_{j} \circ f\right)=k_{j}, \quad j=1, \ldots, n .
$$

From the above proposition and properties of covering multiplicity of holomorphic mappings we have 
Corollary 1.2. Let $f=\left(f_{1}, \ldots, f_{m}\right): \Omega \rightarrow \mathbb{C}^{m}$, and let $k_{j}=\operatorname{ord} f_{j}$, $k_{1} \leq \ldots \leq k_{m}$. Then:

(i) $i_{0}(f) \geq k_{1} \ldots k_{n}$.

(ii) If

$$
i_{0}(f)=k_{1} \ldots k_{n}
$$

then the initial forms in $f_{1}, \ldots$, in $f_{l}$ have no nontrivial zeros in common, where $l=\max \left\{j: k_{j}=k_{n}\right\}$.

(iii) If the forms in $f_{1}, \ldots$, in $f_{n}$ have no common nontrivial zeros, then (4) holds.

Proof. From Proposition 1.1, by the assumptions on the orders $k_{j}$, for the generic $L=\left(L_{1}, \ldots, L_{n}\right) \in \Delta(m, n)$ we have

$$
\text { ord } L_{j} \circ f=k_{j}, \quad j=1, \ldots, n,
$$

and

$$
i_{0}(f)=\mu_{0}(L \circ f) .
$$

Then from the properties of covering multiplicity in the proper case $m=n$ we obtain (i).

(ii) Assume that the forms in $f_{1}, \ldots$, in $f_{l}$ have a nontrivial common zero. Then for the generic $L \in \Delta(m, n)$, the initial forms of the components of the mapping $L \circ f$ have a nontrivial zero in common. Thus, by (5), Proposition 1.1, and the properties of covering multiplicity in the proper case, (4) does not hold.

(iii) Assume that (4) does not hold. Then, by (5), (i) and Proposition 1.1, for the generic $L \in \Delta(m, n)$, the initial forms of the components of $L \circ f$ have a nontrivial zero in common. Let

$$
F=\left(F_{1}, \ldots, F_{n}\right): \Delta(m, n) \times \Omega \ni(L, x) \mapsto L(f(x)) \in \mathbb{C}^{n} .
$$

Then for the generic $L \in \Delta(m, n)$, the forms in $F_{1}(L, x), \ldots$, in $F_{n}(L, x)$ have a nontrivial zero in common. Thus for any $L \in \Delta(m, n)$ the above forms have a nontrivial zero in common. In particular taking $L\left(y_{1}, \ldots, y_{m}\right)=$ $\left(y_{1}, \ldots, y_{n}\right) \in \Delta(m, n)$ we see that in $f_{1}, \ldots$, in $f_{n}$ have a common nontrivial zero. This contradicts the assumption.

COROllary 1.3. Let $f=\left(f_{1}, \ldots, f_{m}\right): \mathbb{C}^{n} \rightarrow \mathbb{C}^{m}$ be a polynomial mapping having an isolated zero at 0 . Let $d_{j}=\operatorname{deg} f_{j}, d_{1} \geq \ldots \geq d_{m}>0$. Then

$$
i_{0}(f) \leq d_{1} \ldots d_{n} .
$$

Proof. By Proposition 1.1, there exists $L=\left(L_{1}, \ldots, L_{n}\right) \in \Delta(m, n)$ such that 0 is an isolated zero of $L \circ f$ and $i_{0}(f)=i_{0}(L \circ f)$. By the definition of $\Delta(m, n)$ we have $\operatorname{deg} L_{j} \circ f \leq d_{j}$ for $j=1, \ldots, n$. Then, by 
Proposition 1.3 of $\left[\mathrm{P}_{3}\right]$ we obtain

$$
i_{0}(f)=\mu_{0}(L \circ f) \leq d_{1} \ldots d_{n}
$$

This gives the assertion.

Let us give a formula for the multiplicity of a holomorphic mapping at an isolated zero. We adopt the definition of the degree of an analytic set $A$ at a point $x$ from $[\mathrm{D}], \S 6$, and denote it by $\operatorname{deg}_{x} A$.

Let $\Omega \subset \mathbb{C}^{n}$ be a neighbourhood of $0 \in \mathbb{C}^{n}$ such that $\bar{\Omega}$ is a compact set. Let $f$ be a holomorphic mapping in some neighbourhood of $\bar{\Omega}$ with values in $\mathbb{C}^{m}$ such that $f^{-1}(0) \cap \bar{\Omega}=\{0\}$. By the Remmert proper mapping theorem $\left(\left[\mathrm{E}_{2}\right]\right.$, V.5.1), $f(\Omega)$ is an analytic set at $0 \in \mathbb{C}^{m}$.

Proposition 1.2. Under the above notations,

$$
i_{0}(f)=\alpha \operatorname{deg}_{0} f(\Omega),
$$

where $\alpha=\limsup _{x \rightarrow 0} \#\left(f^{-1}(f(x)) \cap \Omega\right)$. Moreover, $\alpha$ does not depend on the choice of $\Omega$.

Proof. From the assumption, we have $\eta=\inf _{x \in \partial \Omega}|f(x)|>0$. Let $B=\left\{y \in \mathbb{C}^{m}:|y|<\eta / 2\right\}$, and let $D \subset \Omega$ be the connected component of $f^{-1}(B)$ such that $0 \in D$. Obviously $f^{-1}(0) \cap \bar{D}=\{0\}$. Moreover $f: D \rightarrow B$ is a proper mapping. So, for any $y \in B, \#\left(f^{-1}(y) \cap D\right)<\infty$. By the Remmert proper mapping theorem, $W=f(D)$ is an analytic subset of $B$, and, by Proposition 2 of $\left[\mathrm{E}_{2}\right], \mathrm{V} .7 .2, \operatorname{dim} W=n$. Moreover, the germs of the analytic sets $W$ and $f(\Omega)$ at $0 \in \mathbb{C}^{m}$ are equal, thus

$$
\operatorname{deg}_{0} W=\operatorname{deg}_{0} f(\Omega) .
$$

Since the set $W$ is irreducible, $\left.f\right|_{D}: D \rightarrow W$ is a branched covering. It is easy to see that for any neighbourhood $D^{\prime} \subset D$ of $0 \in \mathbb{C}^{n}$, there exists $\varepsilon>0$ such that for any $|y|<\varepsilon$ we have $f^{-1}(y) \cap D^{\prime}=f^{-1}(y) \cap \Omega$. Thus $\alpha$ is the degree of the covering $\left.f\right|_{D}$.

Let $S$ be the set of singular points of $W$. From the definition of $\operatorname{deg}_{0} W$, there exists a dense subset $\mathcal{U} \subset \mathbf{L}(m, n)$ such that for any $L \in \mathcal{U}$, there exists a neighbourhood $U \subset \mathbb{C}^{n}$ of 0 , and an analytic subset $V \subset U, \operatorname{dim} V<n$, such that for all $z \in U \backslash V$,

$$
\#\left[(W \backslash S) \cap L^{-1}(z)\right]=\#\left[W \cap L^{-1}(z)\right]=\operatorname{deg}_{0} W .
$$

Thus, for any $z \in U, \#\left[(L \circ f)^{-1}(z) \cap D\right] \leq \alpha \operatorname{deg}_{0} W$. Moreover, by the Andreotti-Stoll theorem, there exists an analytic subset $V \subset \Sigma \subset U$, $\operatorname{dim} \Sigma<n$, such that for any $z \in U \backslash \Sigma$,

$$
\#\left[(L \circ f)^{-1}(z) \cap D\right]=\alpha \operatorname{deg}_{0} W .
$$

On the other hand, for any $L \in \mathcal{U}$, there exist connected neighbourhoods $D^{\prime} \subset D$ and $B^{\prime} \subset U$ of $0 \in \mathbb{C}^{n}$ such that $L \circ f: D^{\prime} \rightarrow B^{\prime}$ is proper and 
$(L \circ f)^{-1}(0) \cap \overline{D^{\prime}}=\{0\}$. In consequence,

$$
\mu((L \circ f), 0)=\sup _{z \in B^{\prime}} \#\left[(L \circ f)^{-1}(z) \cap D^{\prime}\right]=\alpha \operatorname{deg}_{0} W .
$$

This, by Theorem 1.1, gives the assertion.

Let, as before, $\Omega \subset \mathbb{C}^{n}$ be a neighbourhood of $0 \in \mathbb{C}^{n}$ and $f: \Omega \rightarrow \mathbb{C}^{m}$ be a holomorphic mapping having an isolated zero at 0 .

THEOREM 1.2. There exists a neighbourhood $D \subset \Omega$ of 0 such that $\left.f\right|_{D}: D \rightarrow f(D)$ is a branched covering, $f^{-1}(0) \cap D=\{0\}$ and

$$
i_{0}(f)=\left.\operatorname{deg}_{0} f(D) \operatorname{deg} f\right|_{D} .
$$

Proof. Since 0 is an isolated zero of $f$, there exists a neighbourhood $K$ of 0 such that $\bar{K} \subset \Omega$ is a compact set and $f^{-1}(0) \cap \bar{K}=\{0\}$. So, as in the proof of Proposition 1.2, there exist neighbourhoods $0 \in D \subset K, 0 \in B \subset$ $\mathbb{C}^{m}$ such that $\left.f\right|_{D}: D \rightarrow B$ is a proper mapping. Thus, $\left.f\right|_{D}: D \rightarrow f(D)$ is a branched covering. Moreover $\alpha=\limsup _{x \rightarrow 0} \#\left(f^{-1}(0) \cap D\right)$ does not depend on the choice of $D$ and $\alpha$ is the degree of the branched covering $\left.f\right|_{D}: D \rightarrow f(D)$. In consequence, by Proposition 1.2, we have the assertion.

2. The local Łojasiewicz exponent. Analogous considerations can be applied to the local Łojasiewicz exponent. Let, as usual, $\Omega \subset \mathbb{C}^{n}$ be a neighbourhood of $0 \in \mathbb{C}^{n}$ and let $f: \Omega \rightarrow \mathbb{C}^{m}$ be a holomorphic mapping having an isolated zero at 0 .

Set

$$
N_{0}(f)=\left\{\nu \in \mathbb{R}: \exists_{C>0, r>0} \forall_{x \in \Omega}|x|<r \Rightarrow|f(x)| \geq C|x|^{\nu}\right\} .
$$

The Eojasiewicz exponent at 0 of the mapping $f$ is defined to be $\inf N_{0}(f)$ and denoted by $\mathcal{L}_{0}(f)$.

Theorem 2.1. Let $n \leq k \leq m$. Then for any $L \in \mathbf{L}(m, k)$ such that 0 is an isolated zero of $(L \circ f)^{-1}(0)$ we have

$$
\mathcal{L}_{0}(f) \leq \mathcal{L}_{0}(L \circ f) .
$$

Moreover, for the generic $L \in \mathbf{L}(m, k)$, the point 0 is an isolated zero of $L \circ f$ and

$$
\mathcal{L}_{0}(f)=\mathcal{L}_{0}(L \circ f) .
$$

Proof. Obviously $m \geq n$. If $m=n$ or $k=m$, then the assertion is obvious. Assume that $m>k \geq n$.

Consider the case $k=n$. Let $L \in \mathbf{L}(m, n)$ be such that 0 is an isolated point of $(L \circ f)^{-1}(0)$. Then there exists $M \in \mathbf{L}(m, m-n)$ such that $\widetilde{L}=$ $(L, M): \mathbb{C}^{m} \rightarrow \mathbb{C}^{m}$ is a linear automorphism of $\mathbb{C}^{m}$. Thus $\mathcal{L}_{0}(f)=\mathcal{L}_{0}(\widetilde{L} \circ f)$. Obviously for $x \in \Omega$ we have $|\widetilde{L} \circ f(x)| \geq|L \circ f(x)|$, so $\mathcal{L}_{0}(\widetilde{L} \circ f) \leq \mathcal{L}_{0}(L \circ f)$. This gives the first part of the assertion in this case. 
Let us prove the second part. Since the considerations have a local character, we may assume that $W=f(\Omega)$ generates an irreducible germ at $0 \in \mathbb{C}^{m}$. Let $C_{0}(W)$ be the tangent cone to $W$ at 0 . Then $\operatorname{dim} C_{0}(W)=\operatorname{dim} W$, so for the generic $L \in \mathbf{L}(m, n)$ we have $C_{0}(W) \cap \operatorname{ker} L \subset\{0\}$, thus 0 is an isolated zero of $L \circ f$. By Sadullaev's theorem ([ $\left[\mathrm{E}_{2}\right]$, VII.7.1), for the generic $L \in \mathbf{L}(m, n)$ there exists $M \in \mathbf{L}(m, m-n)$ such that $(L, M) \in \mathbf{L}(m, m)$ and for any $y \in C_{0}(W)$,

$$
|M(y)| \leq \frac{1}{2}|L(y)|
$$

Thus, diminishing $\Omega$ if necessary, we have for any $x \in \Omega$,

$$
|M(f(x))| \leq|L(f(x))| \text {. }
$$

Therefore $|(L, M) \circ f(x)|=|L \circ f(x)|, x \in \Omega$. This gives

$$
\mathcal{L}_{0}(L \circ f)=\mathcal{L}_{0}((L, M) \circ f)=\mathcal{L}_{0}(f) .
$$

So, we have the assertion in this case.

The last case $n<k<m$ is proved as in Theorem 1.1. This ends the proof.

Since the Łojasiewicz exponent is an invariant with respect to linear automorphisms, from Theorem 2.1 we immediately obtain

Proposition 2.1. For the generic $L=\left(L_{1}, \ldots, L_{n}\right) \in \Delta(m, n)$, the point 0 is an isolated zero of $L \circ f: \Omega \rightarrow \mathbb{C}^{n}$ and

$$
\mathcal{L}_{0}(f)=\mathcal{L}_{0}(L \circ f) .
$$

Moreover, if $k_{j}=$ ord $f_{j}, k_{1} \leq \ldots \leq k_{m}$, then

$$
\operatorname{ord}\left(L_{j} \circ f\right)=k_{j}, \quad j=1, \ldots, n .
$$

By the regular separation exponent of closed sets $X, Y$ in an open subset $D \subset \mathbb{C}^{N}$ at a point $a \in X \cap Y$ we mean a number $\nu>0$ such that there exist $C>0$ and a neighbourhood $B \subset D$ of $a$ such that

$$
z \in B \Rightarrow \varrho(z, X)+\varrho(z, Y) \geq C \varrho(z, X \cap Y)^{\nu},
$$

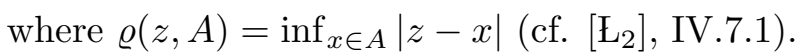

REMARK 2.1. It is easy to observe that the Eojasiewicz exponent of a holomorphic mapping $f: \Omega \rightarrow \mathbb{C}^{m}$ at an isolated zero $0 \in \Omega$ is the smallest regular separation exponent of $\Omega \times\{0\}$ and graph $f$ at $(0,0)$.

REMARK 2.2. From the above remark it follows that the Łojasiewicz exponent of a holomorphic mapping $f=\left(f_{1}, \ldots, f_{m}\right): \Omega \rightarrow \mathbb{C}^{m}$ at an isolated zero $0 \in \Omega$ depends on the ideal $\left(f_{1}, \ldots, f_{m}\right) \mathcal{O}_{0}$ only.

Indeed, let $X=\Omega \times\{0\}, Y=\operatorname{graph} f$. Take any functions $\beta_{i, j}, i, j=$ $1, \ldots, m$, holomorphic in a neighbourhood of $0 \in \mathbb{C}^{n}$ such that the matrix $\left[\beta_{i, j}\right]$ is invertible, and take the biholomorphism $\xi$ as in the proof of Corollary 1.1. Then for $\widetilde{f}=\left[\beta_{i, j}\right] f$ we have $\xi(Y)=\operatorname{graph} \widetilde{f}$. Since the regular 
separation exponent is a biholomorphic invariant (see [ $\left.\mathrm{L}_{2}\right]$, IV.7.1), using Remark 2.1, we see $\mathcal{L}_{0}(f)=\mathcal{L}_{0}(\widetilde{f})$. Hence, as in the proof of Corollary 1.1 we obtain the assertion of the remark.

REMARK 2.3. For the Lojasiewicz exponent, in general, the inclusion $\left(\mathfrak{m}_{0}\right)^{\mathcal{L}_{0}(f)} \subset\left(f_{1}, \ldots, f_{m}\right) \mathcal{O}_{0}$ does not hold. Indeed, if $f=\left(x^{2}, y^{3}\right): \mathbb{C}^{2} \rightarrow \mathbb{C}^{2}$, then $\mathcal{L}_{0}(f)=3$ but $\left(\mathfrak{m}_{0}\right)^{3} \not \subset\left(x^{2}, y^{3}\right) \mathcal{O}_{0}$, because $(x+y)^{3} \notin\left(x^{2}, y^{3}\right) \mathcal{O}_{0}$.

3. Multiplicity and the Łojasiewicz exponent. Using the results of Sections 1 and 2, we may easily generalize some known relations between multiplicity and the Lojasiewicz exponent from the case $m=n$ to the case $m>n$. Namely, we have

Proposition 3.1. Let $\Omega \subset \mathbb{C}^{n}$ be a neighbourhood of 0 and let

$$
f=\left(f_{1}, \ldots, f_{m}\right): \Omega \rightarrow \mathbb{C}^{m}
$$

be a holomorphic mapping having an isolated zero at $0, m \geq n$.

(i) ( $\left[\mathrm{P}_{2}\right]$ for $\left.m=n\right)$. If $g: \Omega \rightarrow \mathbb{C}^{m}$ is a holomorphic mapping such that $\operatorname{ord}(f-g)>\mathcal{L}_{0}(f)$, then 0 is an isolated zero of $g$ and

$$
\mathcal{L}_{0}(f)=\mathcal{L}_{0}(g), \quad i_{0}(f)=i_{0}(g) .
$$

(ii) $\left(\left[\mathrm{P}_{3}\right]\right.$ for $\left.m=n\right)$.

$$
i_{0}(f) \leq\left[\mathcal{L}_{0}(f)\right]^{n}
$$

where $\left[\mathcal{L}_{0}(f)\right]$ denotes the integer part of the number $\mathcal{L}_{0}(f)$. Then

(iii) ([C] for $m=n=2$ and $\left[\mathrm{P}_{3}\right]$ for $\left.m=n\right)$. Let $k_{j}=$ ord $f_{j}, k_{1} \leq \ldots \leq k_{m}$.

$$
k_{n} \leq \mathcal{L}_{0}(f) \leq i_{0}(f)+k_{n}-\prod_{i=1}^{n} k_{i} .
$$

Acknowledgments. I am deeply grateful to Professors Jacek Chądzyński and Tadeusz Krasiński for their valuable comments and advice.

\section{References}

[ATW] R. Achilles, P. Tworzewski and T. Winiarski, On improper isolated intersection in complex analytic geometry, Ann. Polon. Math. 51 (1990), 21-36.

[C] J. Chạdzyński, On the order of isolated zero of a holomorphic mapping, Bull. Polish Acad. Sci. Math. 31 (1983), 121-128.

[CL] S. H. Chang and Y. C. Lu, On $C^{0}$-sufficiency of complex jets, Canad. J. Math. 25 (1973), 874-880.

[D'A] J. D'Angelo, Real hypersurfaces, orders of contact and applications, Ann. of Math. (2) 115 (1982), 615-637.

[D] R. N. Draper, Intersection theory in analytic geometry, Math. Ann. 180 (1969), 175-204. 
[KL] T. C. Kuo and Y. C. Lu, On analytic function germs of two complex variables, Topology 16 (1977), 299-310.

[LJT] M. Lejeune-Jalabert et B. Teissier, Clôture intégrale des idéaux et equisingularité, Centre de Mathématiques, École Polytechnique, 1974.

$\left[\mathrm{E}_{1}\right] \quad$ S. Łojasiewicz, Ensembles semi-analytiques, I.H.E.S., Bures-sur-Yvette, 1965.

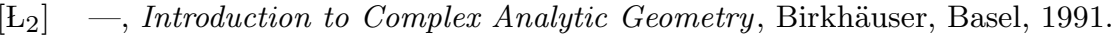

$[\mathrm{M}]$ D. Mumford, Algebraic Geometry I. Complex Projective Varieties, Springer, Berlin, 1976.

$\left[\mathrm{P}_{1}\right]$ A. Płoski, Sur l'exposant d'une application analytique I, Bull. Polish Acad. Sci. Math. 32 (1984), 669-673.

$\left[\mathrm{P}_{2}\right]$-, Sur l'exposant d'une application analytique II, ibid. 33 (1985), 123-127.

$\left[\mathrm{P}_{3}\right]$-, Multiplicity and the Eojasiewicz exponent, in: Banach Center Publ. 20, PWN, 1988, 353-364

[SV] J. Stückrad and W. Vogel, An algebraic approach to the intersection theory, in: The Curves Seminar at Queen's, Vol. II, Queen's Papers Pure Appl. Math. 61, Kingston, Ont., 1982, 1-32.

[Te] B. Teissier, Variétés polaires. I. Invariants polaires des singularités d'hypersurfaces, Invent. Math. 40 (1977), 267-292.

[Tw] P. Tworzewski, Intersection theory in complex analytic geometry, Ann. Polon. Math. 62 (1995), 177-191.

[TW] P. Tworzewski and T. Winiarski, Cycles of zeros of holomorphic mappings, Bull. Polish Acad. Sci. Math. 37 (1989), 95-101.

[V] W. Vogel, Lectures on Results on Bézout's Theorem, notes by D. P. Patil, Lecture Notes, Tata Inst. Fund. Res. Bombay, Springer, 1984.

W] H. Whitney, Tangents to an analytic variety, Ann. of Math. 81 (1965), 496549 .

Faculty of Mathematics

University of Łódź

S. Banacha 22

90-238 Łódź, Poland

E-mail: spodziej@imul.uni.lodz.pl 